\title{
A FRESH LOOK AT GROWTH ASSESSMENT OF INFANTS AND YOUNG CHILDREN IN THE CZECH REPUBLIC IN CONTEXT OF INTERNATIONAL DEVELOPMENTS
}

\author{
Jana Vignerová ${ }^{1}$, Lída Lhotská ${ }^{2}$ \\ 1Department of Biostatistics and Informatics, National Institute of Public Health, Praha, Czech Republic \\ ${ }^{2}$ Regional Coordinator for Europe, International Baby Food Action Network, Geneva, Switzerland
}

\begin{abstract}
SUMMARY
The growth charts of basic body measurements are an important aid for the daily routine practice of paediatricians. Charts for children from birth to 2 years of age form an integral component of a set of such tools for the age spectrum from birth to 18 years of age. The interpretation of growth charts is highly dependent on the data on basis of which the charts were constructed.

In the Czech Republic, the growth reference data have been regularly updated since 1951, in 10-year intervals. These updates are based on data from nation-wide anthropological surveys of children and adolescents. Countries, which do not have their own reference data, have been using for growth assessment the 1977 World Health Organization (WHO) and the National Center for Health Statistics (NCHS) international reference growth charts. These charts, developed for height for age, weight for age, and weight for height, were based for children younger than 2 years on a longitudinal study of North American children.

Over the years, use in practice showed that because of the original criteria used to select the child population studied, these reference growth charts were not suitable for assessing growth of breastfed children. The 1977 reference is based on predominantly artificially, that is formula-fed, child population. The evidence shows that breastfed and artificially fed infants grow differently and that the growth pattern of breastfed children most likely better reflects physiological growth. In 1994, based on the accumulated evidence, the WHO started working on new international standards which would be based on a sample of healthy breastfed children.

The project, the WHO Multicentre Growth Reference Study (MGRS), was carried out from 1997-2003. It focused on collection of growth and development data of 8440 children from different ethnic and cultural groups. The underlying assumption of the project was that in favourable socio-economic conditions and with a recommended level of nutrition and lack of maternal smoking, children's growth is very similar, regardless of their ethnic origin and geographic location. The new growth standard for children $0-5$ years of age will be available early in 2006 . In the Czech Republic, we plan to analyse our national reference against this standard. If considered necessary, a process will be put in place for replacing the Czech reference with the WHO standard.

The objective of this article is to alert health professionals that growth charts currently used in their country may not represent an optimal tool, especially with regard to the assessment of nutritional status for 0 to 2-year-old children, unless already based on data of breastfed children. Generally, the lack of awareness of the difference between the two growth patterns - the breastfed versus artificially fed child - poses a serious problem since a strict interpretation of the growth charts may lead to early supplementation of breastmilk with infant formula and/or premature introduction of complementary foods. Both of these practices tend to lead to premature cessation of breastfeeding, one of the key strategies to improve child health and development.
\end{abstract}

Key words: growth references, growth standards, breastfeeding, child nutrition, anthropometry, child health

Address for correspondence: J. Vignerová, National Institute of Public Health, Department of Biostatistics and Informatics, Šrobárova 48,10042 Praha 10, Czech Republic. E-mail: jvig@szu.cz 
ascertain the health and nutrition status of the Czech child and adolescent population.

Similarly to other countries, the increasing average height of the Czech population necessitated regular updates of growth charts for height and other parameters. The results of the six Nation-wide Anthropological Surveys of Children and Adolescents mentioned above served as basis for these updates. The latest survey was carried out in 2001 (4).

The growth charts of basic body measurements represent an important aid for the daily routine practice of paediatricians. One always has to regard them as a useful tool with the understanding that a child's growth has to be assessed in relation to and together with other factors. The charts cannot be seen as a template which does not require any further consideration.

Growth charts for assessment of length for age and weight for length for children from birth to 2 years of age form an integral component of a set of such tools for the age spectrum from birth to 18 years of age. With their help a paediatrician determines how the child thrives, whether his or her nutrition is adequate.

\section{GROWTH CHARTS RECOMMENDED BY WHO}

In the Czech Republic, as well as in a number of other countries, the growth charts, based on larger-scale, cross-sectional national surveys, describe how children grew at a particular time of the survey. To assist countries which did not have their own reference data, in 1977 the World Health Organization (WHO) and the National Centre for Health Statistics (NCHS) in the USA, recommended international reference growth charts for height for age, weight for age and weight for height. For children younger than 3 years, these charts were developed based on a longitudinal study of a North American population, and for older children, on 3 cross-sectional studies, equally based on an American population $(5,6)$.

Over the years, use in practice showed that these reference growth charts were not suitable for assessing the growth of breastfed children because of the original criteria used to select the child population studied (7). The 1977 reference is based on an infant population that was predominantly artificially, that is formula-fed $(7,8,9,10,11)$.

In the 1990s, the evidence that breastfed and artificially fed infants grow differently kept accumulating and the following questions were increasingly raised: When this 1977 reference is used for growth assessment of breastfed infants, does it not lead to incorrect interpretation and thus to poor nutritional management of breastfed children? Should not the growth pattern of children following the WHO infant and young child feeding recommendations* be regarded as the physiological growth pattern?

To resolve these and similar questions, the WHO Working Group on Infant Growth (10), was given, among others, the task to develop recommendations for use and interpretation of anthropometry for infants. The group focussed at the outset of its work on the known discrepancy that healthy breastfed children of well nourished mothers, who live in good living conditions, manifested apparently 'poorer' growth than artificially fed children from 2-3 months onwards. The slower growth of these healthy infants was in strong contrast with the benefits that are associated with exclusive and continued breastfeeding.

The analysis of data regarding the growth of exclusively and predominantly breastfed children up to 4 months, and who continued to be breastfed for at least 12 months, confirmed that these children deviate negatively when assessed against the current international reference. However, it is most likely that their growth pattern better reflects physiological growth than the trajectory of the 1977 NCHS/WHO international growth reference $(10,14$, 15). Scientific data on the broad impact of benefits inherent in breastfeeding supported this hypothesis.

These findings called for development of new growth charts based on breastfed children, which would describe "how children should grow" and not simply describe how a given population grows, as has been the case of the current NCHS/WHO international reference and many national charts, including the Czech ones $(14,16)$.

\section{THE WHO MULTICENTRE GROWTH REFERENCE STUDY}

A number of smaller studies were needed to build the protocols and implementation plans and strategy of what became known as the WHO Multicentre Growth Reference Study (MGRS) (20). These preliminary studies further confirmed the difference between growth patterns for breastfed and artificially fed infants in the beginning of their life. They showed that up until about 2 months of age, breastfed infants grow in length and gain weight faster than originally projected in the NCHS/WHO reference. Thereafter their growth and weight gain slow down, and until at least the first year they are smaller and leaner.

The principal WHO Multicentre Growth Reference Study itself was carried out in 1997-2003. It focused on the collection of growth and development data of 8,440 children from different ethnic and cultural groups. The underlying assumption of the project was that in favourable socio-economic conditions and with a recommended level of nutrition and absence of maternal smoking, children's growth is very similar, regardless of their ethnic origin and geographic location $(17,18,19)$.

The MGRS took place in six countries on six continents: Brazil, Ghana, India, Norway, Oman, and the USA. It combined the longitudinal study of children from birth to 24 months of age with a cross-sectional study of children 18 to 71 months. Criteria for the selection of the subpopulations, from which the children for the study were chosen included: favourable socio-economic conditions to growth; low mobility of the studied population; at least $20 \%$ of mothers willing to follow the feeding recommendations; and access to breastfeeding support (e.g. Baby-Friendly Hospital Initiative, existence of breastfeeding support groups).

Criteria for the selection of individual children for the study were the following: absence of health, economic or environmental

\footnotetext{
*Recommendation of the World Health Assembly, resolution 54.2, May 2001: six months of exclusive breastfeeding followed by continued breastfeeding for 2 years or beyond with introduction of safe and appropriate complementary foods from completed 6th month onwards (12). The government of the Czech Republic, a WHO Member State, has also a responsibility to ensure compliance with the International Code of Marketing of Breastmilk Substitutes and subsequent relevant resolutions of the World Health Assembly, and for their implementation into the national legislative system (13). The requirements regarding the composition and labelling of infant foods have been already incorporated in the intimation of the Ministry of Health (Law Digest CR, 54/2004).
} 
constraints on growth; adherence to MGRS feeding recommendations; absence of maternal smoking; single birth at term (259-294 days); standard paediatric care and absence of significant newborn morbidity (20).

The criteria for compliance with feeding recommendations were the following:

- Exclusive or predominant breastfeeding for at least 4 months (120 days) $* *$

- Introduction of complementary foods by the age of 6 months (180 days)

- Partial breastfeeding to be continued for at least 12 months (365 days)

In the longitudinal part of the study, the children's measurements were taken at home, in total 21times (at 1,2, and 6 weeks; thereafter monthly; and every two months from 1 to 2 years). In addition to the anthropometric measurements and data about motor development, information about socio-economic, demographic, environmental characteristics as well as prenatal factors and nutritional practices was collected (20).

Data collection and most of the data analysis have been completed. Thus the 1977 NCHS/WHO international reference, based on the North American population, will soon be replaced by new, truly international standards for length/height, weight and weight for length/height. Furthermore, a new standard for triceps and subscapular skinfolds, head and arm circumferences, and body mass index will also be issued.

The longitudinal component of the MGRS will also allow the development of standards for monitoring the growth velocity of various body characteristics, which will make it possible for paediatricians to diagnose under- or over-nutrition before children become undernourished or obese.

Simultaneously, reference data on motor development will be published, providing a unique link for assessing physical growth together with motor development.

The new standards will be available for children up to 5 years of age. However, WHO clearly stated that this effort needed to be expanded to older children.

The standards for length/height for age, weight for age, weight for length/height and BMI for age are expected to be available before the end of 2005, followed by the rest of the standards by the end of 2006.

It is, however, clear that all the effort must not end with a simple substitution of the 1977 NCHS/WHO growth charts by the new ones. The international effort has to be translated into national actions. Practices employed in growth monitoring by health professionals will have to be revisited as a whole. It is not only a matter of use and interpretation of the charts, but also a question of effective prevention and of implementation of interventions to treat inadequate growth at both individual as well as population levels. The new charts will be particularly important for the monitoring of the rapidly spreading prevalence of child obesity. It will also facilitate promotion of breastfeeding as a key strategy to improve child health and development and as an important practice to prevent obesity (21).

\section{GROWTH CHARTS FOR THE CZECH CHILD POPU- LATION}

The tradition of anthropological surveys ranks the Czech Republic among those countries in which use of the growth charts has been a natural element of preventive care for children and adolescents. Currently, after a period of less widespread use, a successful effort was made to reinstate the charts as an integral part of the Health and Vaccination Card of Children and Adolescents. These charts have been presented to parents and the wider public in numerous publications. This campaign led to increased awareness of the existence and purpose of the charts among parents.

The recent update of the charts was based on the results of the 6th Nationwide Anthropological Survey of Children and Adolescents (2001) (1). This survey showed that while the height of the Czech children and adolescents had not changed in any significant way compared to the 1991 data (with the exception of adolescent boys), the Body Mass Index of school children had significantly increased. Thus a cessation of the secular trend in height was noted, while values for weight continue to increase. This finding had a bearing on the update of the charts: only charts for height were updated. The 1991 growth charts for BMI and weight for height remained valid, as updating these charts would have resulted in an undesirable shift in the charts' percentiles towards higher values, negatively affecting the assessment of overweight and obesity.

\section{DISCUSSION}

Although the decision to continue using the BMI charts from 1991, as explained above, is likely to have a positive practical impact on detection and prevention of overweight and obesity, it may not be the sole adjustment of the charts needed. The development of the new WHO standard for infants and young children calls for a fresh and more in-depth look at the Czech reference for this age group (22). Although the new standards are not yet available, we can hypothesize, that our Czech percentile growth reference charts, just like the 1977 NCHS/WHO reference, manifest a rather different trajectory than is foreseen to be the one of the forthcoming WHO Child Growth Standards. There is no nation-wide collection of data on exclusive or predominant and continued breastfeeding in the Czech Republic, which would be using WHO indicators and could thus reliably present the infant feeding situation in the country. The nation-wide Anthropological Surveys are no exception. Although information about breastfeeding was collected during the 6th Nationwide Anthropological Survey 2001, it is not available for all children, and specific data about exclusivity of breastfeeding and the form of artificial and complementary feeding is - despite all the efforts - very unreliable. It is therefore not possible to establish what proportion of children enrolled in the survey and on whose measurements the growth charts are based, were breastfed for an extended period

**Exclusive breastfeeding: the infant has received only breastmilk from its mother or a wetnurse, or expressed breastmilk, and no other liquids or solids with the exception of drops or syrups consisting of vitamins, mineral supplements, or medicines. Predominant breastfeeding: The infant's predominant source of nourishment has been breastmilk. However, the infant may also have received water and water-based drinks; fruit juice; oral rehydration salts solutions; drop and syrop forms of vitamins, minerals and medicines; and ritual fluids in limited quantities. With the exception of fruit juice and sugar water, no foodbased fluid is allowed under this definition (20). 
and what was the breastfeeding pattern. However, the data suggest a steadily improving yet still suboptimal pattern of infant feeding practices in the country. It can be safely assumed that the Czech growth reference has been constructed based on the data of children whose nutrition did not follow WHO recommendation. It is thus possible that an inadequate model of growth charts is being used for those 0 to 2 year-old Czech children who are breastfed for an extended period of time.

\section{CONCLUSION}

In the introduction to this article we noted that the growth charts have to be seen as a useful aid, not a tool that takes decisions for us. Growth of any child has to be assessed in connection with other factors. Paediatricians with sufficient practice are well aware of this and thus able to evaluate whether the decrease in weight gain or slower growth of a breastfed child is already critical or whether it follows an appropriate growth pattern, although not reflected in the growth chart. Generally, however, the lack of awareness of the differences between the two growth patterns - the breastfed versus the artificially fed child - remains a serious problem. The most common outcome of strict interpretation of the charts results in incorrect nutritional recommendations to parents, resulting in early supplementation of breast milk with infant formula and/or premature introduction of complementary foods. Both of these practices more often than not lead to premature cessation of breastfeeding.

In all such cases, the charts correctly depicting growth of breastfed children will help remove the hesitation and uncertainties of both health professionals and parents.

The growth charts are, after a period during which their use declined, again gradually finding their way into routine paediatric practice and are increasingly understood by parents. It is therefore important to alert professionals to the fact that the Czech growth charts for children up to 2 years may not be providing the level of information needed and that they will be assessed for the extent of their bias as soon as the new WHO standards will have been published. Health professionals and parents will be informed about the outcome of the comparison. If considered necessary, a process through which the "old" Czech charts will be replaced by the new WHO standard will need to be put in place to ensure that both practitioners and parents have at their disposal an optimal tool to help them assess growth and development of infants and young children.

\section{Acknowledgement}

The 6th Nationwide Anthropological Survey 2001 is supported by the Internal Grant Agency of the Ministry of Health of the Czech Republic No NJ 6792-3/2001 and NR 7857-3/2004

\section{REFERENCES}

1. Matiegka J. Somatology of school children. Prague: Publishing House of the Czech Academy of Sciences and Arts; 1927. (In Czech.)

2. Prokopec M. Forty years of monitoring child growth in the Czech Republic: methodologies, outcomes and comparisons. In: Eiben OG, editor. Auxology '94: children and youth at the end of the 20th century: Invited and selected papers from the 7th International Conggress of Auxology; 1994 Jun; Szombathely, Hungary. Humanbiologia budapestinensis, 25. Budapest: ITC Plantin; 1994. p. 231-40.

3. Lhotská L, Bláha P, Vignerová J, Roth Z, Prokopec M. Vth nation-wide anthropological survey of children and adolescents 1991 (Czech Republic): evaluation of parents' questionnaire. Prague: National Institute of Public Health; 1995. (In Czech.)

4. Bláha P, Vignerová J, Riedlová J, Kobzová J, Krejčovský L. VIth nation-wide anthropological survey of children and adolescents 2001. Cesk Pediatr. 2003; 58(12):766-70. (In Czech.)

5. Dibley MJ, Goldsby JB, Staehling NW, Trowbridge FL. Development of normalized curves for the international growth reference: historical and technical considerations. Am J Clin Nutr. 1987 Nov;46(5):736-48.

6. Hamill PVV. NCHS growth curves for children: birth-18 years, United States. Vital and health statistics: Series 11, Data from the National Health Survey; no 165, DHEW publication; (PHS) 78-1650. Hyattsville: U.S. Department of Health, Education, and Welfare; 1977.

7. Cole TJ, Paul AA, Whitehead RG. Weight reference charts for British long-term breastfed infants. Acta Pediatr. 2002;91(12):1296-300.

8. de Onis M, Onyango AW. The Centers for Disease Control and Prevention 2000 growth charts and the growth of breastfed infants. Acta Paediatr. 2003 Apr;92(4):413-9.

9. de Onis M, Victoria CG. Growth charts for breastfed babies. J Pediatr (Rio J). 2004 Mar-Apr;80(2):85-7.

10. WHO Working Group on Infant Growth. An evaluation of infant growth. Geneva: World Health Organization; 1994.

11. The optimal duration of exclusive breastfeeding. Report of an expert consultation; 2001 Mar 28-30; Geneva, Switzerland. Geneva: World Health Organization; 2002.

12. Infant and young child nutrition. WHA Resolution 54.2. Fifty-fourth World Health Assembly; 2001 May 14-22; Geneva, Switzerland. Geneva: World Health Organization; 2001.

13. Schneidrová D. Tools to protect breastfeeding against inappropriate marketing of breastmilk substitutes. Cas Cesk Lek. 2002;74(2):26-7. (In Czech.)

14. Garza $\mathrm{C}$, de Onis $\mathrm{M}$. Rationale for developing a new international growth reference. Food Nutr Bull. 2004 Mar;25(1 Suppl 1):S5-14.

15. WHO Working Group on Infant Growth. An evaluation of infant growth: the use and interpretation of anthropometry in infants. Bull World Health Organ. 1995;73(2):165-74.

16. de Onis M, Garza C, Habicht JP. Time for a new growth reference. Pediatrics. 1997 Nov;100(5):E8.

17. Habicht JP, Martorell R, Yarbrough C, Malina RM, Klein RE. Height and weight standards for preschool children. How relevant are ethnic differences in growth potential? Lancet. 1974 Apr 6;1(7858):611-4.

18. Ulijaszek SJ. Between-population differences in human growth. In: Ulijaszek SJ, Johnston FE, Preece MA, editors. The Cambridge encyclopedia of human growth and development. Cambridge: University Press; 1998. p. 361-4.

19. WHO Working Group on the Growth Reference Protocol and WHO Task Force on Methods for the Natural Regulation of Fertility. Growth patterns of breastfed infants in seven countries. Acta Pediatr. 2000 Feb;89(2):215-22.

20. de Onis M, Garza C, Victora CG, Onyango AW, Frongillo EA, Martines J. The WHO Multicentre Growth Reference Study: planning, study design, and metodology. Food Nutr Bull. 2004;25(1 Suppl 1):S15-26.

21. Owen CG, Martin RM, Whincup PH, Smith GD, Cook DG. Effect of infant feeding on the risk of obesity across the life course: a quantitative review of published evidence. Pediatrics. 2005 May;115(5):1367-77.

22. Vignerová J, Lhotská L. Growth charts for breastfed children and growth assessment of infants and young children in the Czech Republic. Cesk Pediatr. 2005;60(11):612-6. (In Czech). 